\title{
N6-methyladenosine modification of circCUX1 confers radioresistance of hypopharyngeal squamous cell carcinoma through caspase 1 pathway
}

\author{
Ping Wu', Xing Fang ${ }^{1}$, Yalan Liu', Yaoyun Tang $\mathbb{1}^{1}$, Wei Wang ${ }^{1}$, Xin $\mathrm{Li}^{1}$ and Yuhua Fan ${ }^{1}$
}

\begin{abstract}
Hypopharyngeal squamous cell carcinoma (HPSCC) is one of the most common malignant tumors in otolaryngology head and neck surgery and is one of the worst prognostic malignant tumors. Endogenous circular RNA (circRNA) is more stable than mRNA, microRNA (miRNA), and long non-coding RNA (LnCRNA) in exosomes, plasma, and urine, and participates in gene expression regulation to perform different functions. Therefore, circRNA is expected to become a biomarker and therapy target for many tumors. However, the expression and function of circRNA regulated by N6methyladenosine (m6A) are still unclear in HNSCC. In this study, we demonstrated that a specific circRNA, circCUX1, was upregulated in HPSCC patients who are resistant to radiotherapy and predicts poor survival outcome. We further found that methyltransferase like 3 (METTL3) mediated the m6A methylation of circCUX1 and stabilizes its expression. Knockdown circCUX1 promotes the sensitivity of hypopharyngeal cancer cells to radiotherapy. In addition, circCUX1 binds to Caspase 1 and inhibits its expression, resulting in a decrease in the release of inflammatory factors, thereby developing tolerance to radiotherapy. Our findings indicate that circCUX1 is a potential therapeutic target for radiotherapy tolerance in HPSCC patients.
\end{abstract}

\section{Introduction}

Head and neck squamous cell carcinoma (HNSCC), including laryngeal cancer, oral cancer, nasopharyngeal cancer, hypopharyngeal cancer, has many primary sites and pathological types and more than $90 \%$ of head and neck tumors are squamous cell carcinoma ${ }^{1}$. Patients with early stage HNSCC can obtain good curative effects by surgery or radiotherapy. However, the prognosis of advanced patients is often unsatisfactory ${ }^{2,3}$. Understanding the pathogenesis of HNSCC is crucial for improving the diagnosis and cure rate of patients. Hypopharyngeal squamous cell carcinoma (HPSCC) is one of the most common malignant tumors in

\footnotetext{
Correspondence: Yaoyun Tang (csuwoodpink@126.com)

${ }^{1}$ Department of Otorhinolaryngology Head \& Neck Surgery; Province Key

Laboratory of Otolaryngology Critical Diseases, Xiangya Hospital, Central South University, Changsha 410008, China
}

Edited by $\mathrm{G}$. Calin otolaryngology head and neck surgery ${ }^{4}$. It has the characteristics of hidden location, strong infiltration, easy submucosal spread, and multicentric growth of the primary lesion. Because there are no obvious symptoms in the early stage, the lack of specific signs and reliable diagnostic measures, most HPSCC patients are already in the advanced stage when they are diagnosed ${ }^{5}$. In addition, chronic inflammation is often a key factor in cancer development. As the head and neck area is prone to exposure to factors causing irritation and inflammation of the squamous epithelium, it might therefore be plausible that chronic inflammation also might be a major cause for the development of $\mathrm{HNSCC}^{6,7}$. there are abundant lymphatic vessels in the laryngopharyngeal area, which are prone to cervical lymph node metastasis and extracapsular spread, and invade surrounding tissues and organs, such as carotid arteries and other important blood vessels ${ }^{8}$. HPSCC is one of the worst

\section{(c) The Author(s) 2021}

(c) (i) Open Access This article is licensed under a Creative Commons Attribution 4.0 International License, which permits use, sharing, adaptation, distribution and reproduction c. in any medium or format, as long as you give appropriate credit to the original author(s) and the source, provide a link to the Creative Commons license, and indicate if changes were made. The images or other third party material in this article are included in the article's Creative Commons license, unless indicated otherwise in a credit line to the material. If material is not included in the article's Creative Commons license and your intended use is not permitted by statutory regulation or exceeds the permitted use, you will need to obtain permission directly from the copyright holder. To view a copy of this license, visit http://creativecommons.org/licenses/by/4.0/. 
prognostic malignant tumors. Therefore, it is an urgent issue to propose new diagnostic methods and less toxic and side effects treatment methods based on molecular biology.

Circular RNAs (circRNAs) are a special type of RNA molecule, mostly circRNA molecules without $5^{\prime}$ and $3^{\prime}$ ends formed by reverse splicing of more than one exon, which are abundant in eukaryotic cells ${ }^{9}$. CircRNAs have high degree of conservation and tissue specificity ${ }^{10}$. At present, the mechanism of regulating the expression of circRNA is not completely clear. Studies have found that the production and function of non-coding RNAs such as microRNA (miRNA), long-chain non-coding RNA (lncRNA), and circRNA (circRNA) are regulated by N6-methyladenosine (m6A) modification ${ }^{11}$. M6A refers to the methylation modification that occurs on the sixth nitrogen atom of adenine, and it is widely present in the RNA of many eukaryotes. Current research shows that m6A-related proteins mainly include three types of methyltransferases that catalyze the formation of $\mathrm{m} 6 \mathrm{~A}$, demethylases that remove $\mathrm{m} 6 \mathrm{~A}$ modifications, and methyl-binding proteins that recognize $\mathrm{m} 6 \mathrm{~A}$ modifications ${ }^{12}$. Recent studies have found that there are also m6A modifications on circRNA, which are jointly regulated by the demethylase FTO and the methyltransferase METTL3 ${ }^{13}$. In the tumor tissues of patients with primary non-small cell lung cancer, the positive expression rate of METTL3 is higher than that of normal tissues around the cancer, and the level of METTL3 is associated with the patient's tumor size, depth of invasion, and lymph node metastasis ${ }^{14}$. After the use of small interference RNA interference technology to reduce the level of METTL3 in lung cancer cells, the level of m6A modification on RNA is reduced, and malignant phenotypes such as cell proliferation, migration, and invasion are inhibited ${ }^{14}$. However, the expression and function of circRNA regulated by $\mathrm{m} 6 \mathrm{~A}$ are still unclear in HNSCC.

Endogenous circRNA participates in gene expression regulation through different mechanisms and performs different functions ${ }^{15}$. CircRNA is more stable than mRNA, miRNA, and long non-coding RNA (lncRNA) in exosomes, plasma, urine, and saliva ${ }^{16}$. Therefore, circRNA is expected to become a biomarker for disease diagnosis. Studies have found that circRNA CDR1as promote the growth and glucose metabolism of nasopharyngeal carcinoma cells by sponging miR- $7^{17,18}$. The latest study found that circRNA 0000285 increased significantly in the serum and tissues of patients with nasopharyngeal carcinoma, and affected the sensitivity of radiotherapy in patients with nasopharyngeal carcinoma ${ }^{19}$. CircRNA microarray analysis of HPSCC obtained 2392 differential circRNA molecules ${ }^{20}$. However, the role of these circRNAs in HPSCC is not fully clear.

In this study, we demonstrated that circCUX1 was upregulated in HPSCC patients who were resistant to radiotherapy and predicted a poor survival outcome. We further found that METTL3-mediated the m6A methylation of circCUX1 and stabilized its expression. Knockdown circCUX1 promoted the sensitivity of hypopharyngeal cancer cells to radiotherapy. CircCUX1 bound to caspase 1 and inhibits its expression, resulting in a decrease in the release of inflammatory factors, thereby developing tolerance to radiotherapy. Our findings indicate that circCUX1 is a potential therapeutic target for radiotherapy tolerance in HPSCC patients.

\section{Materials and methods \\ Tumor sample collection}

This study was approved by the ethics committee of Xiangya Hospital of Central South University. Before the operation, written informed consent was signed by the patient. Inclusion criteria: (1) First diagnosed in our hospital and confirmed pathologically as HPSCC; after diagnosis, radiotherapy was performed (2) Re-examination in our hospital 3 months after the end of radiotherapy, the imaging data was complete; At 6 months, the cervical lymph nodes did not completely disappear. The neck lymph node dissection specimens were performed, and the patient was pathologically diagnosed as a head and neck tumor and neck metastasis. From 2015 to 2019, human tumor tissues were collected from 78 patients with HPSCC collected from our hospital, including 68 males and 10 females, aged 28-76 years, with a median age of 51 years. And 60 cases of adjacent tissues during the same period were used as the control group. According to WHO's radiotherapy response assessment criteria for solid tumors $^{21,22}, 3$ months after the end of radiotherapy, patients with HPSCC were divided into radiotherapysensitive group $(N=40)$ and radiotherapy-resistant group $(N=38)$. All patients receive regular follow-up. The overall survival (OS) time is from the date of surgery to the date of death or the last follow-up of the survivor. The disease-free survival (DFS) is the survival time from the first radiotherapy to the tumor recurrence or death.

\section{Cell culture}

The head and neck tumor cell lines SCC-4, SCC-9, SCC-15, CLA-27, SAS, Tca-8113, and Fadu cell lines, and human oral keratinocyte (HOK) cells were purchased from the American Type Culture Collection (ATCC). All cells were cultured in RPMI-1640 containing 10\% fetal bovine serum medium (Invitrogen, Carlsbad, USA). All cells were cultured in a humidified incubator at $37^{\circ} \mathrm{C}$ and $5 \%$ carbon dioxide.

\section{RNA-seq analysis}

Five pairs of HPSCC tumor samples and matched paracancerous samples (included 3 pairs of sensitive specimens and 2 pairs of resistant specimens) were randomly selected for RNA sequencing analysis, which was 


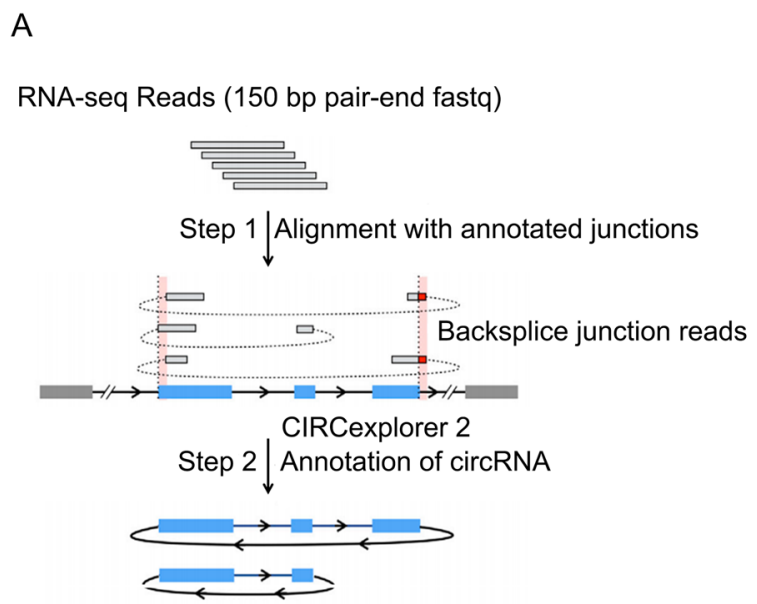

C

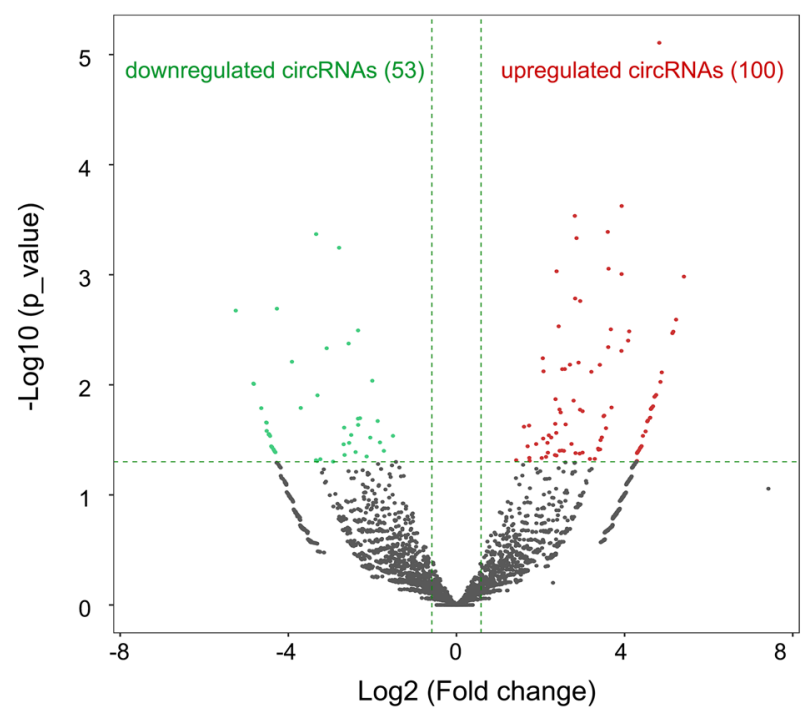

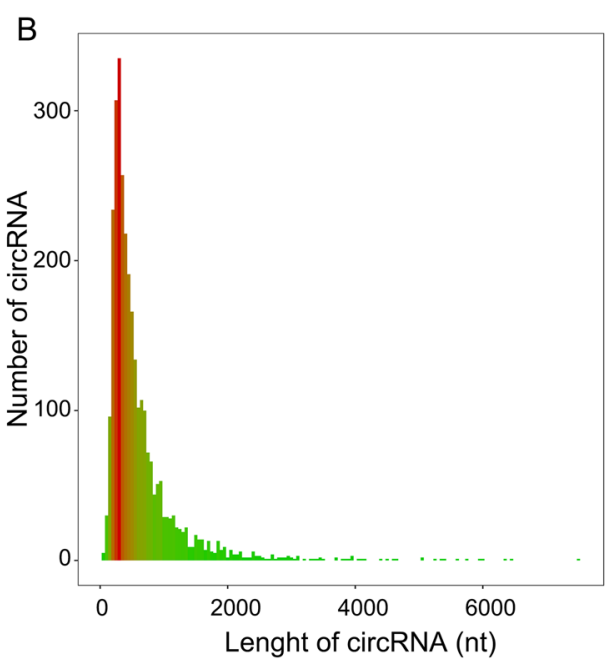

D

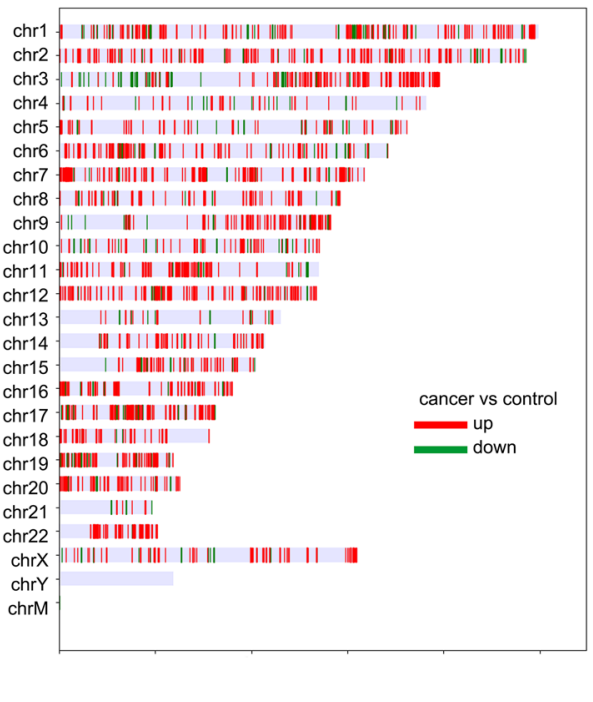

Fig. 1 RNA sequencing reveals the dysregulated circRNAs in HPSCC. A Flowchart illustrating the RNA sequencing and circRNA annotation. B circRNAs length distribution. C Volcano plot showed the downregulated circRNA and upregulated circRNA. D Downregulated and upregulated circRNA distribution in chromosome. chM chromosome mitochondria.

completed by Aksomics (Shanghai, China). NanoDrop ND-1000 was used to determine the RNA concentration. One to two micrograms of total RNA from each sample was used to construct the RNA sequencing library. Briefly, after the total RNA sample is enriched with oligo dT (rRNA removal), using KAPA Stranded RNA-Seq Library Prep Kit (Illumina) constructs the library. The doublestranded cDNA synthesis in the library construction process using the dUTP method combined with the subsequent high-fidelity PCR polymerase to make the final RNA sequencing library chain specific. The constructed library was identified by Agilent 2100 Bioanalyzer for library quality, and the library was quantified by qPCR method. The mixed library of different samples is sequenced using the Illumina HiSeq 4000 sequencer. Solexa pipeline version 1.8 (Off-Line Base Caller software, version 1.8) software was used for image processing and base identification. FastQC software evaluates the sequencing quality of reads after removing adapters. $\mathrm{R}$ software Ballgown was used to calculate FPKM at gene level and transcript level, and calculate the difference in gene level and transcript level expression respectively, and screen the differentially expressed genes between samples or groups. New gene/transcript prediction is assembled by StringTie for each sample, and compared with official annotation information, obtained through Ballgown calculation. CircRNAs were compared to the reference genome by STAR software, and CIRCexplorer2 was used 
for Backsplice junction reads detection, reads count statistics, and $\mathrm{R}$ software edge $\mathrm{R}$ was used for differential expression calculation. The sequencing flowchart is shown in Fig. 1A. The threshold was set to be 1.5 -fold difference, $p$ value $\leq 0.05$ and intra-group CPM average $\geq 100$ to screen for differentially expressed circRNA.

\section{RNA quantitative real-time polymerase chain reaction}

TRIzol kit was used to extract total RNA from tissues and cells. UV spectrophotometer was used to detect RNA quality. QuantiTect reverse transcription kit (Cat No. 205313, Qiagen, USA) was used to reverse transcription to cDNA. Real-time polymerase chain reaction (RT-PCR) was performed using SYBR Green Master Mix (Bio-Rad, USA) on ABI7900HT (Applied Biosystems, CA, USA). The reaction conditions are: $95^{\circ} \mathrm{C}$ for $10 \mathrm{~s} ; 95^{\circ} \mathrm{C}$ for $5 \mathrm{~s}$, $60^{\circ} \mathrm{C}$ for $30 \mathrm{~s}, 72^{\circ} \mathrm{C}$ for $30 \mathrm{~s}, 40$ cycles. $\beta$-actin was used as an internal reference gene. The primer sequences are listed in supplementary Table 1.

\section{Actinomycin D and RNase R treatment}

Fadu cells were seeded into a six-well plate. After $24 \mathrm{~h}$, when reached $70 \%$ confluence, the cells were treated with $5 \mu \mathrm{g} / \mathrm{ml}$ actinomycin and collected at the designated time point. For RNase R treatment, total RNA $(2 \mu \mathrm{g})$ and $3 \mathrm{U} /$ $\mu \mathrm{g}$ RNase R (Epicenter Technologies, Madison, WI, USA) were incubated at $37{ }^{\circ} \mathrm{C}$ for $15 \mathrm{~min}$. After actinomycin D or RNase R treatment, the RNA expression levels of circCUX1 and CUX1 mRNA were analyzed by qRT-PCR.

\section{RNA fluorescence in situ hybridization (FISH)}

The sequence of the oligonucleotide modified probe circCUX1 was synthesized by Sangon Biotech (Shanghai, China). The paraformaldehyde-fixed cell slides were washed in PBS, and treated with RNase $\mathrm{R}$ at $37^{\circ} \mathrm{C}$ for $15 \mathrm{~min}$, and then fixed again. After dehydration with ethanol, the probes were hybridized overnight at $37^{\circ} \mathrm{C}$ in a dark box. The slides were washed twice with $50 \%$ formamide for $5 \mathrm{~min}$ each. The slides were incubated with Alexa Fluor 488 reagent (Proteintech, Wuhan, China) for $30 \mathrm{~min}$, and sealed with a parafilm containing DAPI. A fluorescence microscope (OLYMPUS, Japan) was used to obtain the pictures. The probe sequence is as follows: circCUX1: 5'-Biotin labeled-UCCCGACACACUCGAGU CUAACUACGUGACUCAUUUCUUCGUUC - 3 ' .

\section{RNA interference (RNAi) and transfection}

The siRNA used to knock out circCUX1 and METTL3 was designed and synthesized from Ribobio (Guangzhou, China). The siRNA target sequence used to construct the lentivirus as: circCUX1 siRNA 1\#, TGTGAGGAGTCT AACTAGCACTGAGT; circCUX1 siRNA 2\#, GACAAA GAGATTGATGCACTGA; METTL3 siRNA, GCACAU CCUACUCUUGUAATT. Fadu or SCC-9 cells are seeded into a 24-well culture plate at a density of $4 \times 10^{4}$ cells per well to ensure that the cell confluence can reach about $60 \%$ during transfection. The medium was replaced with $500 \mu \mathrm{l}$ fresh complete medium, and added with $6 \mu \mathrm{l}$ each well of the lentiviral particle stock solution and negative control virus to the 24-well culture plate, accompanied with $0.5 \mu \mathrm{l}$ polybrene (final concentration $5 \mathrm{ng} / \mathrm{ml}$ ), The cells were cultured in $37^{\circ} \mathrm{C}$ incubator for $48 \mathrm{~h}$, and collected for subsequent experiments.

\section{ELIZA}

After the cells are transfected, the cell culture supernatant is collected. The human IL-1 $\beta$ and IL-18 kit (cat no. KE00021, KE00025; Proteintech Group, Wuhan, China) were used to analyze the IL- $1 \beta$ and IL-18 concentrations using follow the manufacturer's instructions.

\section{Cell growth inhibition assay}

Fadu or SCC-9 cells were transfected with lentiviruses for $48 \mathrm{~h}$ and then seeded into a 6 -well $\left(5 \times 10^{5}\right.$ per well $)$ plate and cultured overnight. A medical electron linear accelerator was used for X-ray irradiation (distance $100 \mathrm{~cm}$, irradiation area $20 \mathrm{~cm} \times 20 \mathrm{~cm}$, single irradiation, irradiation dose of $0,1,2,4,6$ Gy). After irradiation, cells were continually cultured for $24 \mathrm{~h}$. The cell survival rate was detected by Cell Counting Kit 8 (CCK-8) (Abcam, China) according to the manufacturer's instructions. CCK- 8 allows very convenient assays by utilizing highly water-soluble tetrazolium salt. WST-8 [2-(2-methoxy-4nitrophenyl)-3-(4-nitrophenyl)-5-(2,4-disulfophenyl)-2Htetrazolium, monosodium salt] produces a water-soluble formazan dye upon reduction in the presence of an electron mediator. CCK-8 allows sensitive colorimetric assays for the determination of the number of viable cells in cell proliferation assays. The median lethal dose (LD50) was calculated.

\section{RNA pull-down}

The biotin-labeled circCUX1 probe was mixed with total cell protein and incubated for $24 \mathrm{~h}$ at $4{ }^{\circ} \mathrm{C}$ to make circCUX1 and protein form a circCUX1-protein complex, and then the magnetic beads (MEGA clear Kit, Ambion) were used to adsorb the complex. The protein in the capture complex was analyzed by Western blotting, using sodium dodecyl sulfate (SDS)-polyacrylamide gel (PAGE) electrophoresis. The proteins were transferred on polyvinylidene fluoride (PVDF) membrane. The membrane was incubated with $5 \%$ non-fat milk for $1 \mathrm{~h}$ at $37^{\circ} \mathrm{C}$ and incubated with a primary antibody METTL3 (diluted 1:1000, Abcam) overnight at $4{ }^{\circ} \mathrm{C}$. After washed with PBS, the membrane was incubated with the secondary antibody for $2 \mathrm{~h}$ at room temperature. A SuperSignal West Atto Ultimate Sensitivity Substrate (cat no. A38555, ThermoFisher, USA) was used to protein blot detection in the gel imaging system. 
RNA-binding protein immunoprecipitation (RIP)

A Magna RIPTM RNA-Binding Protein Immunoprecipitation Kit from Millipore was used for RNA immunoprecipitation in accordance with the kit instructions. Briefly, after $48 \mathrm{~h}$ transfection, Fadu cells were harvested and lysed with RIP lysis buffer on ice for $10 \mathrm{~min}$. After centrifugation, the supernatant was incubated with $30 \mu \mathrm{l}$ Protein-A/G magnetic beads (Roche, USA) and antibody (METTL3, $10 \mu \mathrm{g}$, Abcam; METTL14, $10 \mu \mathrm{g}$, Abcam) at $4{ }^{\circ} \mathrm{C}$ overnight. The complex was then centrifuged and then washed 3 times with washing buffer. Immunoprecipitated RNA was used in qRT-PCR analysis.

\section{Luciferase reporter gene determination}

The wild type (WT) or mutant (Mut) Caspase $1 \mathrm{cDNA}$ fragments of the target binding site predicted by circCUX1 were inserted into the luciferase report gene pGL3 (Promega) to construct a recombinant vector. Fadu cells were co-transfected with circCUX1 (800 ng) or circCUX1 siRNA $(20 \mathrm{pmol})$ and WT or Mut Caspase 1 $(20 \mathrm{pmol})$ in a 24 -well culture plate $\left(1 \times 10^{5}\right.$ cells per well). The transfection process was carried out using Lipofectamine 2000 system. After $48 \mathrm{~h}$ of transfection, the cells were collected, and the luciferase activity was detected by a Thermo Scientific Vanquish system (Thermo Scientific, USA).

\section{Statistical analysis}

SPSS 16. 0 software was used to carry on statistical analysis to the data. All experimental data were performed independently three times, and the data was expressed as mean \pm standard deviation (mean $\pm \mathrm{SD}$ ). The obtained data were compared using one-way analysis of variance and Bonferroni's post-correction Student's $t$ test. The difference was statistically significant with $P<0.05$.

\section{Results}

Analysis of dysregulated circRNA in HPSCC tissue

We performed high-throughput RNA sequencing in five pairs of HPSCC and matched adjacent non-tumor tissue samples. The experimental process is shown in Fig. 1A. The length of circRNA expressed in HPSCC tissues is mainly within $2000 \mathrm{nt}$ (Fig. 1B). Screening by the following criteria: RNA expression was upregulated or downregulated, and average normalized fold change $\geq 2$. We found 53 downregulated circRNAs and 100 upregulated circRNAs (Fig. 1C); Table 1 lists the top ten upregulated circRNAs. Except for Y chromosome and mitochondria, dysregulated circRNAs were found in all other chromosome (Fig. 1D).

We used qPCR to verify the expression of differential circRNA in Table 1 in ten pairs of HPSCC and matched adjacent non-tumor tissue samples, including four pairs of sensitive specimens and six pairs of resistant specimens that was different from the panel of five matched pairs of clinical specimens in initial screening. We found that except for the expression of circSIPA1L2, the expression of other circRNAs in tumor tissues was significantly higher than that in adjacent tissues, especially the expression of circCUX1 (Fig. 2A). Therefore, we choose circCUX1 (circRNA_0081609, hg 19, chr7: 101713618-101848450, exon 4 - exon 20) for further analysis. We tested the expression of circCUX1 in HOK and seven head and neck tumor cell lines. Compared with HOK cells, the expression of circCUX1 in head and neck tumor cell lines was significantly increased, especially in the hypopharyngeal carcinoma cell line Fadu (Fig. 2B).

To study the clinical significance of circCUX1 in hypopharyngeal cancer patients, a cohort of 78 hypopharyngeal cancer patients with survival data was included. Since radiotherapy is an important treatment for patients with head and neck tumors, and radiotherapy tolerance is an important prognostic predictor,

Table 1 Top ten upregulated circRNAs in HPSCC tissues.

\begin{tabular}{|c|c|c|c|c|c|c|}
\hline circRNA & Locus & Gene Name & Length & Fold Change & $P$ value & $Q$ value \\
\hline hsa_circ_0114428 & chr1:91403041-91447927:- & ZNF644 & 3705 & 42.57 & 0.00103 & 0.28247 \\
\hline hsa_circ_0081607 & chr7:101559394-101838883:+ & CUX1 & 1192 & 37.39 & 0.00255 & 0.46150 \\
\hline hsa_circ_0008255 & chr16:16200594-16205439:+ & $\mathrm{ABCC} 1$ & 344 & 35.72 & 0.00328 & 0.46150 \\
\hline hsa_circ_0008992 & chr11:77090285-77091039:- & PAK1 & 249 & 35.20 & 0.00339 & 0.46150 \\
\hline hsa_circ_0006051 & chr22:25771779-25771975:- & LRP5L & 196 & 29.53 & 0.00770 & 0.62274 \\
\hline hsa_circ_0112397 & chr1:232649602-232651354:- & SIPA1L2 & 1752 & 28.90 & 0.00940 & 0.71638 \\
\hline hsa_circ_0057608 & chr2:197777605-197786910:- & PGAP1 & 586 & 26.91 & 0.01235 & 0.72194 \\
\hline hsa_circ_0008912 & chr12:72020039-72022817:- & ZFC3H1 & 491 & 26.19 & 0.01284 & 0.72194 \\
\hline hsa_circ_0006358 & chr4:169812072-169825057:+ & PALLD & 607 & 25.12 & 0.01609 & 0.72194 \\
\hline hsa_circ_0095454 & chr11:16339991-16362798:- & sox6 & 449 & 25.09 & 0.01612 & 0.72194 \\
\hline
\end{tabular}




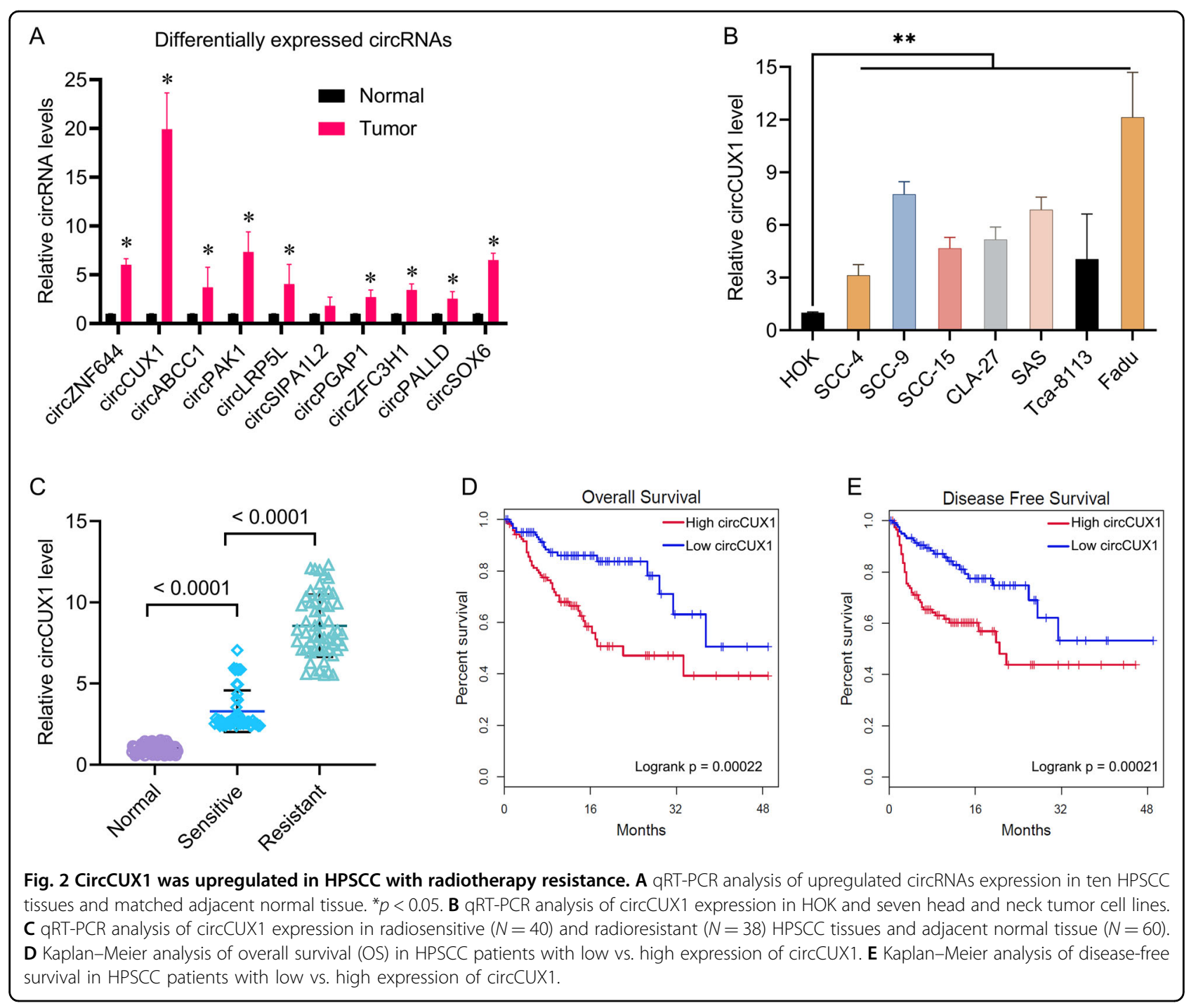

we further investigated whether the expression of circCUX1 is related to radiotherapy tolerance in patients with hypopharyngeal carcinoma. The 78 patients included 38 radiotherapy-resistant patients and 40 radiotherapy-sensitive patients, and 60 adjacent nontumor tissues were collected as controls. We found that compared with the control group, the expression of circCUX1 was significantly upregulated in cancer tissues, especially in patients with radiotherapy tolerance (Fig. 2C). In addition, circCUX1 was induced by irradiation in Fadu and SCC-9 cells (Supplementary Fig. 1). We next used the mean value of circCUX1 expression as the cutoff value to divide the patients into high circCUX1 group and low circCUX1 group. Through Kaplan-Meier analysis, we found that high expression of circCUX1 indicated poor OS and poor DFS (Fig. 2D, E). We further analyzed the correlation between the expression of circCUX1 and the clinical characteristics of patients with HPSCC. As shown in Table 2, the expression of circCUX1 was significantly correlated with primary tumor size $(p<0.001)$, lymph node metastasis $(p=0.0121)$, distant metastasis $(p=0.0394)$, and TNM stage $(p=0.0219)$. There was no significant correlation between the expression of circCUX1 and age $(p=$ 0.2643). Univariate and multivariate Cox regression analysis found that circCUX1 was an independent factor affecting the survival of patients with HPSCC (Univariate analysis: $\mathrm{HR}, 3.87$; 95\% CI, 1.94-6.24, $p=0.0063$; multivariate analysis: $\mathrm{HR}, 2.83$; $95 \% \mathrm{CI}, 1.74-5.02, p=$ 0.0167) (Tables 2, 3, and 4), suggesting that circCUX1 can be used as a biomarker for HPSCC.

\section{Characterization of circCUX1 in hypopharyngeal carcinoma cells \\ CircCUX1 is derived from exons 4 to 20 (hg 19, chr7: 101713618-101848450, GRCh37/hg 19) in the cut-like}


Table 2 Association of HPSCC characteristics and circCUX1 expression.

\begin{tabular}{lllc}
\hline & High circCUX1 & Low circCUX1 & $P$ value \\
\hline N, \% & $45(57.7)$ & $33(42.3)$ & \\
Age, year, mean (SD) & $48.8(15.3)$ & $54.3(10.4)$ & 0.2643 \\
Tumor size, cm, mean (SD) & $2.6(1.4)$ & $3.8(1.2)$ & $<0.001$ \\
Lymphatic metastasis, N & & & 0.0121 \\
No & 18 & 23 & \\
N1-3 & 27 & 10 & 0.0394 \\
Distant metastasis, N & & & \\
No & 22 & 24 & 0.0219 \\
Yes & 23 & 9 & \\
TNM stage, N & & & \\
I or II & 15 & 13 & \\
III or IV & 30 & & \\
\hline
\end{tabular}

Table 3 Univariate analysis of prognostic factors of HPSCC.

\begin{tabular}{lll}
\hline Variable & Hazard ratio (95\% Cl) & $\boldsymbol{p}$ value \\
\hline Tumor size ( $\geq 3$ vs. $<3)$ & $2.24(1.66-5.42)$ & 0.0213 \\
Lymphatic metastasis (N1-3 vs. N0) & $3.14(2.24-5.86)$ & 0.0162 \\
Distant metastasis (Yes vs. No) & $5.21(3.53-7.72)$ & 0.0031 \\
TNM stage ((III-IV vs. I/II) & $4.56(2.67-8.25)$ & 0.0056 \\
circCUX1 (High vs. Low) & $3.87(1.94-6.24)$ & 0.0063 \\
\hline
\end{tabular}

homeobox 1 (CUX1) locus, which has been described in neuroblastoma by $\mathrm{Li}$ et $\mathrm{al}^{23}$. We aim to study the characterization of circCUX1 in HPSCC. Divergent and convergent primers were used to check the specificity of the primers and the correctness of the circularization site ( $5^{\prime}$ exon 20 to $3^{\prime}$ exon 4 ) by RT-PCR (Fig. 3A, B). RNase R-resistant exonuclease digestion confirmed that circCUX1 has a circRNA structure (Fig. 3C). Actinomycin $\mathrm{D}$ is a transcription inhibitor that can be used to analyze the half-life of RNA. After actinomycin D treatment, qRT-PCR analysis showed that the half-life of circCUX1 exceeded $24 \mathrm{~h}$, while the half-life of linear transcript approximately was $8 \mathrm{~h}$ (Fig. 3D), indicating that circCUX1 is more stable in hypopharyngeal cancer cells. Further, fluorescence in situ hybridization (FISH) revealed that circCUX1 was mainly located in the cytoplasm (Fig. 3E). Together, these results reveal that circCUX1 is a rich and stable circRNA expressed in hypopharyngeal cancer cells.
Table 4 Multivariate analysis of independent prognostic factors of HPSCC.

\begin{tabular}{lll}
\hline Variable & Hazard ratio (95\% Cl) & $\boldsymbol{p}$ value \\
\hline Tumor size ( $\geq 3$ vs. <3) & $1.97(1.27-3.57)$ & 0.0352 \\
Lymphatic metastasis (N1-3 vs. N0) & $2.79(1.65-4.68)$ & 0.0265 \\
Distant metastasis (Yes vs. No) & $4.35(2.04-6.87)$ & 0.0125 \\
TNM stage ((III/IV vs. I/II) & $3.16(2.07-6.15)$ & 0.0086 \\
CircCUX1 (High vs. Low) & $2.83(1.74-5.02)$ & 0.0167 \\
\hline
\end{tabular}

\section{METTL3-mediated m6A modification stabilizes the expression of circCUX1}

The recent researches show the important role of m6A epigenetic modification in tumor radiotherapy. We want to know whether circCUX1 contains m6A methylation. Through methylated RNA immunoprecipitation (MeRIP) analysis, we precipitated a known m6A-containing RNAs, RARA, and circCUX1 also contains abundant m6A methylation (Fig. 4A), confirming the m6A methylation in circCUX1. METTL3 is a predominant methyltransferase for m6A modification and plays an oncogenic role in tumor formation and progression ${ }^{24}$. We performed RNAbinding protein immunoprecipitation (RIP) analysis and proved that compared with the control IgG, METTL3 antibody-precipitated complexes enriched the expression of circCUX1, while METTL14 could not enrich circCUX1 (Fig. 4B). We performed an RNA pull-down experiment to further verify the combination of METTL3 and circCUX1 (Fig. 4C). In addition, we want to know that the m6A methyltransferase METTL3 whether affect circCUX1 activity. Silencing of METTL3 significantly inhibited the expression of circCUX1 (Fig. 4D, E), and shortened the half-life of circCUX1 RNA compared with scramble (METTL3 siRNA: $28 \mathrm{~h}$ vs. scramble: $57 \mathrm{~h}$, Fig. 4F). These results were also confirmed in SCC-9 cells (Supplementary Fig. 2). The above results suggest that METTL3 stabilizes the expression of circCUX1 through m6A methylation modification.

\section{Silencing circCUX1 promotes the sensitivity of hypopharyngeal cancer cells to radiotherapy}

We knocked down the expression of circCUX1 in Fadu and SCC-9 cells by siRNA transfection. We found that siRNA $2 \#$ transfection had higher efficiency in inhibiting circCUX1 expression than siRNA 1\# transfection (Fig. 5A), while both siRNA 1\# and siRNA 2\# transfection did not alter the expression of CUX1 mRNA (Supplementary Fig. 3). Our results showed that circCUX1 knockdown significantly inhibited the cell viability, and increased the release of inflammatory factors IL-1 $\beta$ and IL-18 in Fadu and SCC-9 cells without 

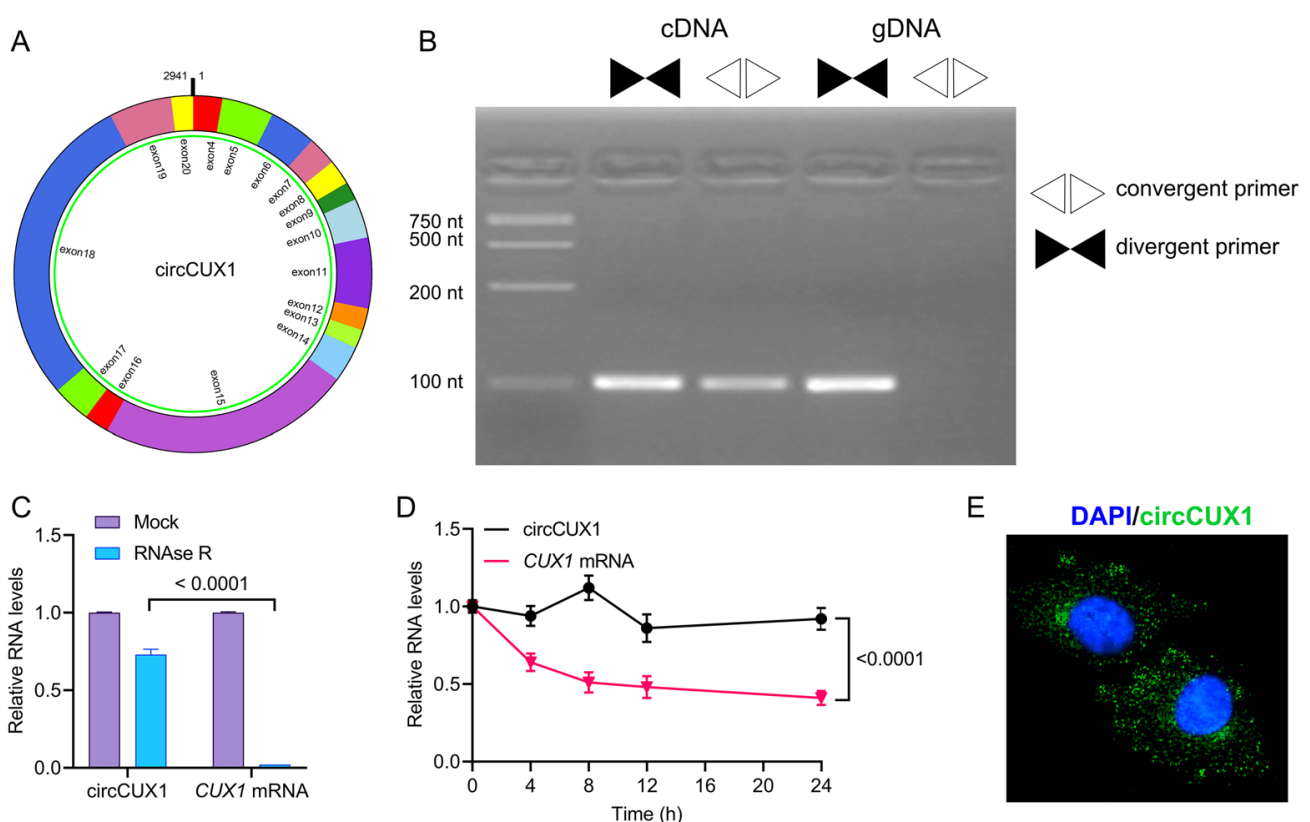

Fig. 3 Characterization of circCUX1 in HPSCC. A The circCUX1 structure. B qRT-PCR products with divergent primers showing circularization of circCUX1. cDNA complementary DNA, gDNA genomic DNA. C qRT-PCR analysis for the expression of circCUX1 and CUX1 mRNA after treatment with RNase $R$ in Fadu cells. D qRT-PCR analysis for the expression of circCUX1 and CUX1 mRNAs after treatment with Actinomycin D at the indicated time points in Fadu cells. E RNA fluorescence in situ hybridization for circCUX1. Nuclei were stained with DAPI.

irradiation exposure (Supplementary Fig. 4). We then studied the role of circCUX1 in radiotherapy by determining the cell viability through a CCK-8 experiment. The results showed that circCUX1 knockdown significantly reduced the LD50 of Fadu and SCC-9 cells, and the reduction of LD50 was positively associated with the decrease of circCUX1 levels (SCC-9 cells: LD50 in scramble: $4.1 \mathrm{~Gy}$, LD50 in siRNA 1\#: $3.1 \mathrm{~Gy}$ vs. LD50 in siRNA 2\#: $2.2 \mathrm{~Gy}, p<0.05$; Fadu cells: LD50 in scramble: 4.9 Gy, LD50 in siRNA 1\#: 4.25 Gy vs. LD50 in siRNA 2\#: $2.95 \mathrm{~Gy}, p<0.05)$, suggesting that circCUX1 knockdown promoted the sensitivity of cells to radiotherapy (Fig. 5B). In addition, circCUX1 knockdown also significantly increased the release of inflammatory factors IL- $1 \beta$ and IL-18, and the levels of IL- $1 \beta$ and IL18 in siRNA 2 \# group were higher than in siRNA 1\# group (Fig. 5C).

\section{CircCUX1 regulates Caspase 1 mRNA}

Studies have shown that Caspase-1 can directly cleave IL-1 $\beta$ precursor (pro-IL-1 $\beta$ ) and IL-18 precursor (proIL-18) to make them active IL- $1 \beta$ and IL-18, and release them to extracellular area becomes an inducer of inflammatory response and participates in the progression of inflammation-related tumors. Knockdown of circCUX1 increased the release of IL- $1 \beta$ and IL-18. We want to know whether circCUX1 can regulate the expression of caspase 1 . We confirmed that circCUX1 knockdown can increase the expression of caspase 1 (Fig. 6A). AREsite analysis (http://rna.tbi.univie.ac.at/ AREsite2) and BLAST analysis suggested that the UUAUUU site inside of circCUX1 could directly bind to the $3^{\prime}$ UTR of caspase 1 with AAUAAA motif. Therefore, we further investigated whether circCUX1 is essential for the stability of caspase1 mRNA. We constructed WT caspase1-3'UTR (caspase1-WT) or Mut $3^{\prime}$ UTR (caspase1-Mut). Overexpression of circCUX1 significantly inhibited the activity of WT caspase $1-3^{\prime}$ UTR luciferase reporter gene, but did not affect the activity of Mut caspase 1-3'UTR luciferase reporter gene (Fig. 6B). In addition, knockdown of circCUX1 greatly increased luciferase activity of WT caspase 1 (Fig. 6C), but not caspase1-Mut. Our RNA pull-down analysis also confirmed the interaction between circCUX1 and caspase 1 (Fig. 6D).

To further reveal the clinical significance of circCUX1 regulating caspase 1 in hypopharyngeal carcinoma, we checked the expression level of caspase1 and analyzed the correlation between caspase 1 and circCUX1. We found that compared with normal tissues adjacent to cancer, caspase 1 was significantly reduced in hypopharyngeal cancer tissues, especially in radiotherapy-resistant tissues (Fig. 6E). It is worth noting that the expression of caspase 1 in hypopharyngeal carcinoma tissue was negatively correlated with the expression of circCUX1 $(r=-0.4643$, $p=0.0003, N=78$, Fig. 6F). 


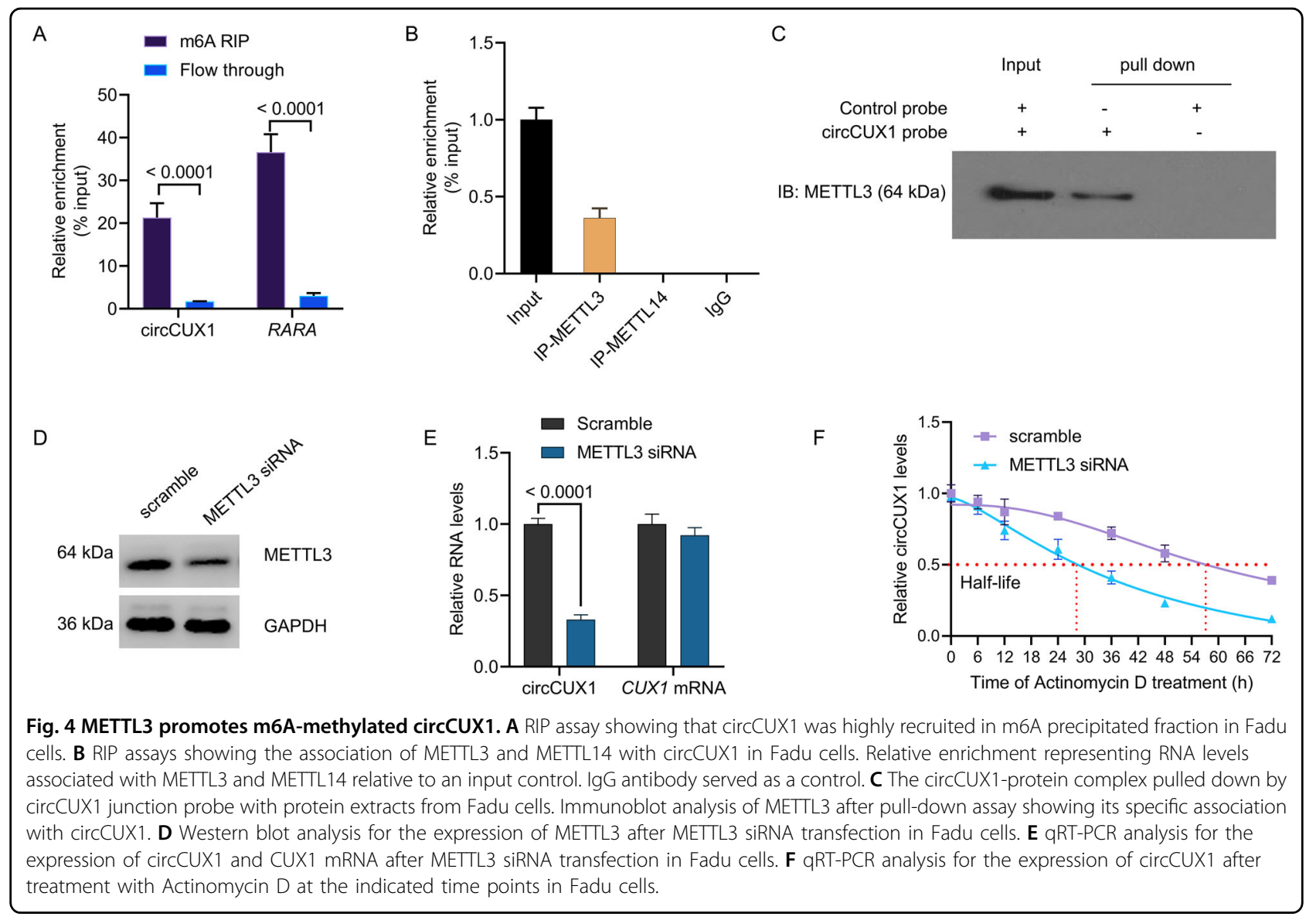

\section{CircCUX1 promotes radiotherapy resistance of hypopharyngeal carcinoma through caspase 1}

Then, we investigated whether circCUX1 depends on the caspase1 pathway in the progression of radiotherapy tolerance in hypopharyngeal carcinoma. Our results showed that knockdown of caspase1 significantly reversed the release of inflammatory factors IL- $1 \beta$ and IL-18 mediated by circCUX1 knockdown (Fig. 7A, B). In addition, knockdown of caspase1 could significantly reverse circCUX1 knockdown-mediated radiotherapy sensitivity (Fig. 7C, D). These data indicate that circCUX1-promoted hypopharyngeal cancer cell radiotherapy resistance depends on the caspase 1 pathway.

\section{Discussion}

In this study, we first time demonstrated the important role of circCUX1 in the radiotherapy resistance of HPSCC. We proved that HPSCC patients with high expression of circCUX1 had a poor prognosis. We observed a significant increase in the expression of circCUX1 in HPSCC tissues and found that circCUX1 might be used as a diagnostic and prognostic indicator for patients with HPSCC resistant to radiotherapy.
In addition, we found that METTL3-mediated the m6A methylation of circCUX1 and stabilized its expression. Importantly, circCUX1 bound to caspase1 mRNA and inhibited its expression, thereby inhibiting caspase 1 mediated inflammation. The discovery of circRNA provides new research ideas for the occurrence, development, treatment, and prevention of tumors, and the high tissue specificity and stability of circRNA make it suitable as a new biomarker ${ }^{25}$. Many researchers report that circRNAs are significantly expressed in tumors, and affect the development of tumors ${ }^{11}$. Although many studies have confirmed the biological functions of circRNA in physiological and pathological processes, the specific mechanism of circRNA in HPSCC needs to be further revealed. M6A is the most abundant modified form of eukaryotic mRNAs, which affects almost all stages of mRNA metabolism, including RNA folding, splicing, translation, and decay, and other RNA modifications ${ }^{13}$. M6A not only affects mRNA metabolism, but also participates in the regulation of non-coding $\mathrm{RNA}^{26}$. More and more evidence show that the dynamic changes of m6A modification provide a new direction for the formulation of tumor radiotherapy and chemotherapy strategies ${ }^{27}$. M6A 


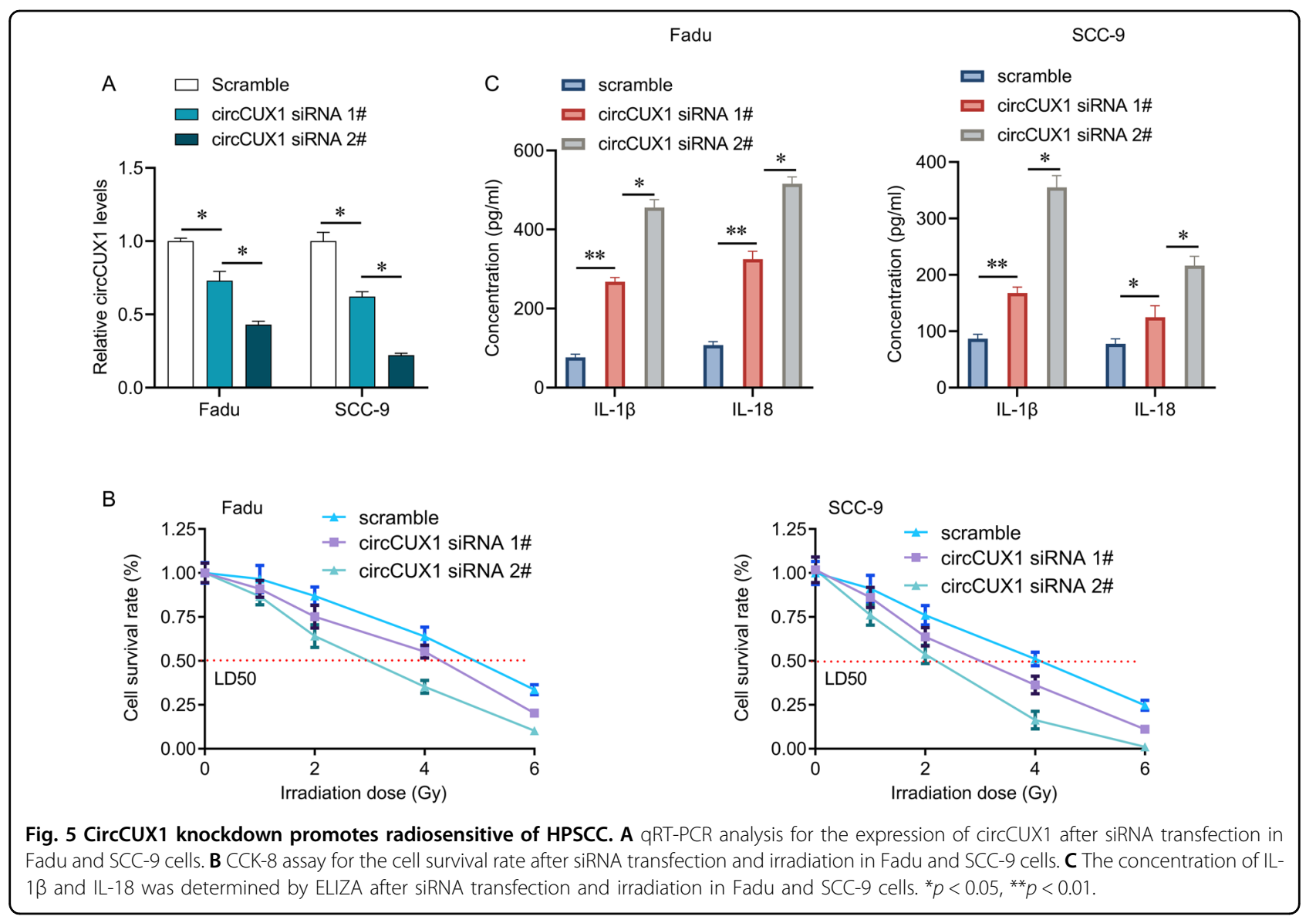

methyltransferase [such as METTL3, METTL14, and METTL16] participates in the progression of malignant tumors by read, write, or erase m6A on bound RNA ${ }^{24}$. The latest research also fully proves the important role of m6A epigenetic modification in tumor radiotherapy and chemotherapy. Studies have found that m6A methylation in RNA can regulate the DNA damage response induced by ultraviolet rays, thereby affecting tumor chemotherapy and radiotherapy sensitivity. METTL3 and METTL14 in the m6A methyltransferase component are expected to become new targets for tumor radiotherapy and chemotherapy sensitization, and METTL3 can promote the chemotherapy and radiotherapy sensitivity of pancreatic cancer cells ${ }^{24}$. Our study provides the first evidence that the stable expression of circCUX1 in the cytoplasm is dependent on the METTL3-mediated m6A modification. The above studies emphasize the importance of m6A as a cancer modification mechanism. The dynamic changes of m6A levels have different regulatory functions on cancer cells. By revealing previously unidentified tumor gene regulatory mechanisms, it provides a basis for exploring the pathogenesis of some tumors and seeking new potential therapeutic targets, and provides new ideas for tumor epigenetic modification mechanisms and tumor gene targeted therapy.

CircRNAs exert their biological functions three mechanisms: (1) regulating gene expression, transcription, and splicing levels; (2) translating into protein to play a role; (3) acting as miRNA sponges through their binding sites to regulate the activity of miRNAs on other target genes ${ }^{28}$. For example, circCUX1 played an oncogenic role in neuroblastoma through targeting miR-165 p/DMRT2 signaling cascade ${ }^{29}$. In addition, circCUX1 binds to EWS RNA-binding protein 1 to facilitate its interaction with MYC-associated zinc finger protein, resulting in neuroblastoma progression ${ }^{23}$. Herein, we found that circCUX1 can bind to caspase 1 mRNA and inhibit its expression. Interestingly, circCUX1 regulates the inflammatory response of tumor cells to radiotherapy through caspase 1. Since Rudolf Virchow first proposed the hypothesis that "cancer originates from chronic inflammation", more and more studies have confirmed that the occurrence and development of tumors are inseparable from inflammation ${ }^{30}$. Epidemiologic evidence suggests that $\sim 25 \%$ of all human cancer worldwide is associated with chronic inflammation, chronic infection, or both ${ }^{6,31}$. Inflammation, chronic inflammation 


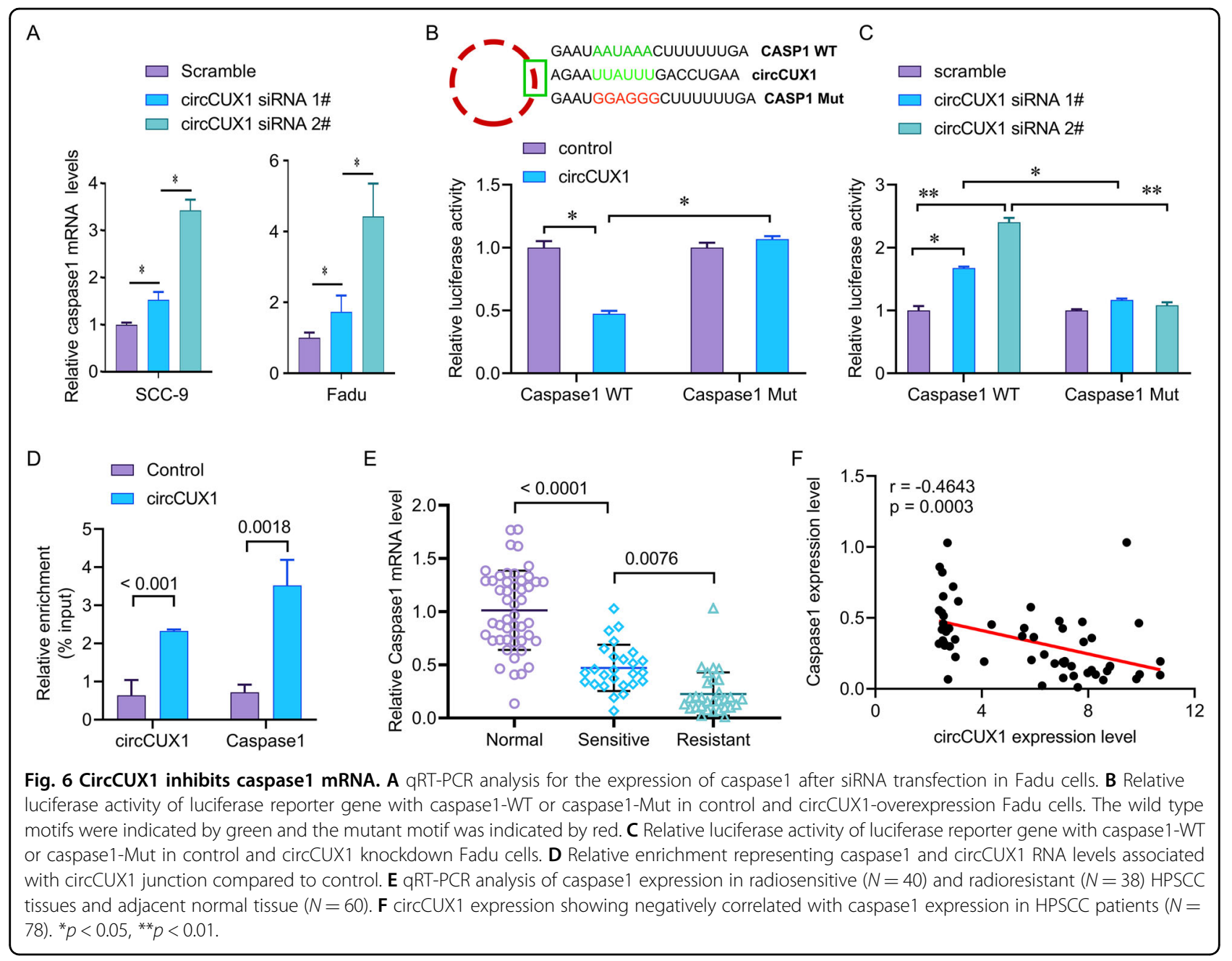

has become the seventh major biological feature of malignant tumors, and such tumors are also called inflammation-related tumors ${ }^{32}$. Studies have shown that many inflammatory cell infiltration and aggregation of inflammatory factors are one of the significant pathological features of head and neck malignant tumors, suggesting that uncontrollable inflammation plays an important role in the occurrence and development of head and neck malignancies ${ }^{33}$. Caspase- 1 is the first Cysteinyl aspartate specific proteinase (Caspase) family member found in mammals, also known as Interleukin$1 \beta$ converting enzyme (ICE) ${ }^{34}$. Recent studies have found that Caspase-1 not only plays a key role in the inflammatory response, but is also closely related to a new type of programmed cell death-pyroptosis ${ }^{35}$. Caspase- 1 can participate in the regulation of the tumor inflammatory microenvironment in many ways, and affect the occurrence, development, invasion, and metastasis of tumors $^{36}$. The expression level of Caspase- 1 may be related to the degree of inflammation in tumor tissues. Caspase- 1 and its mediated pro-inflammatory substances may play a very important role in the occurrence and development of inflammation-related tumors ${ }^{37,38}$. In many chronic inflammation-related tumors, there is usually the activation of inflammasomes ${ }^{39}$. The activated Caspase- 1 in this process can directly cleave the IL- $1 \beta$ precursor (pro-IL-1 $\beta$ ) and IL-18 precursor (pro-IL-18) makes it become active IL- $1 \beta$ and IL-18, and is released to the outside of the cell to become an inducer of inflammation. As an important inflammation-related molecule, Caspase- 1 can participate in the regulation of tumor inflammation microenvironment in a variety of ways. In the process of interaction between malignant tumor cells and their microenvironment among them, Caspase- 1 actively induces tumor cell programmed death and anti-tumor immune surveillance ${ }^{40}$.

\section{Conclusion}

We find that circCUX1 is an important oncogenic circRNA, which may be used as a prognostic biomarker for radiotherapy resistance of HPSCC. METTL3-mediated m6A modification plays a key role in stabilizing the 
A

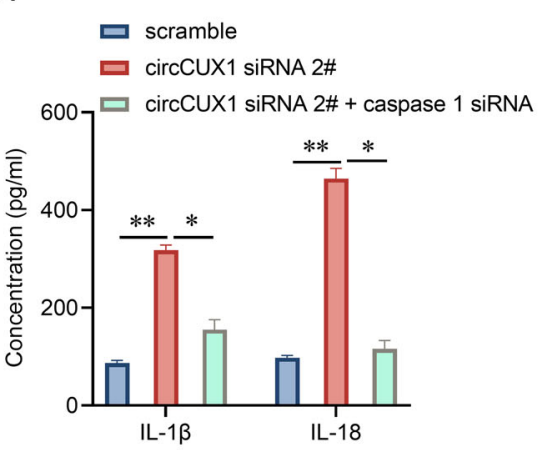

C

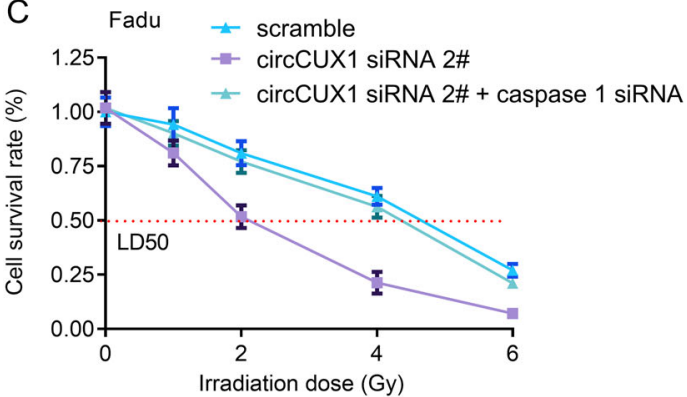

B

SCC-9

acramble

circCUX1 siRNA 2\#

$\square$ circCUX1 siRNA 2\# + caspase 1 siRNA
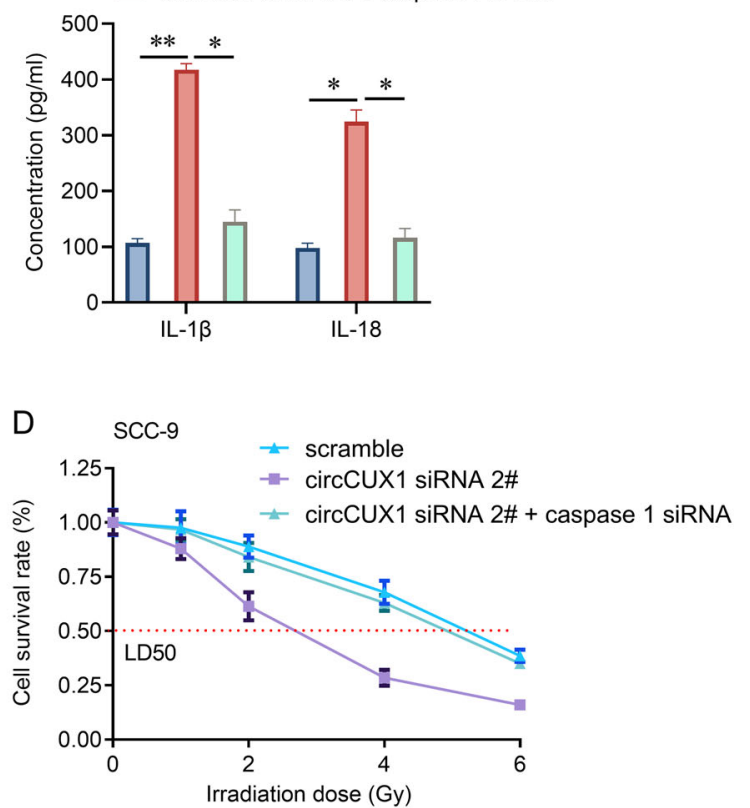

Fig. 7 CircCUX1 confers radioresistance of HPSCC through caspase1 pathway. A, B The concentration of IL-1 $\beta$ and IL-18 was determined by ELIZA after siRNA transfection and irradiation in Fadu (A) and SCC-9 (B) cells. C, D CCK-8 assay for the cell survival rate after siRNA transfection and irradiation in Fadu (C) and SCC-9 (D) cells.

expression of circCUX1, thereby inhibiting the expression of caspase 1 and conferring the radiotherapy resistance of HPSCC. Importantly, our findings provide potential therapeutic targets for patients with HPSCC who are resistant to radiotherapy.

\section{Author contributions}

P.W. and Y.T. conceived the study and participated in the study design, performance, coordination, and manuscript writing. P.W., X.F., Y.L., Y.T., W.W., X. $L$., and Y.F. performed the research. All authors have read and approved the final manuscript. In Fig. 1, P.W. and Y.T. generated the data and assembled the figure. In Fig. 2, P.W., X.F., Y.L., and Y.T. generated the data and labeled the image, W.W. and X.L. assembled the figure. In Fig. 3, X.F. and Y.L. generated the data and labeled the image, X.L. assembled the figure. In Fig. 4, Y.L. and Y.T. generated the data and labeled the image, P.W. assembled the figure. In Fig. 5, X.F. generated the data and labeled the image, P.W. assembled the figure. In Fig. 6, P.W. generated the data and labeled the image, Y.T. assembled the figure. In Fig. 7, W.W. generated the data and labeled the image, X.L. assembled the figure.

\section{Funding statement}

The authors received no specific funding for this work.

\section{Data availability}

The datasets used and/or analyzed during the current study are available from the corresponding author on reasonable request.

\section{Conflict of interest}

The authors declare no competing interests.

\section{Ethics statement}

This study was approved by the ethics committee of Xiangya Hospital of Central South University and was performed in accordance with the Declaration of Helsinki. Written informed consent was obtained from each subject.

\section{Publisher's note}

Springer Nature remains neutral with regard to jurisdictional claims in published maps and institutional affiliations.

Supplementary information The online version contains supplementary material available at https://doi.org/10.1038/s41419-021-03558-2.

Received: 9 October 2020 Revised: 18 February 2021 Accepted: 22 February 2021

Published online: 19 March 2021

\section{References}

1. Leemans, C. R., Snijders, P. J. F. \& Brakenhoff, R. H. The molecular landscape of head and neck cancer. Nat. Rev. Cancer 18, 269-282 (2018).

2. Marur, S. \& Forastiere, A. A. Head and neck squamous cell carcinoma: update on epidemiology, diagnosis, and treatment. Mayo Clin. Proc. 91, 386-396 (2016).

3. Specenier, P. \& Vermorken, J. B. Optimizing treatments for recurrent or metastatic head and neck squamous cell carcinoma. Expert Rev. Anticancer Ther. 18, 901-915 (2018).

4. Cramer, J. D., Burtness, B., Le, Q. T. \& Ferris, R. L. The changing therapeutic landscape of head and neck cancer. Nat. Rev. Clin. Oncol. 16, 669-683 (2019).

5. Gregoire, V., Grau, C., Lapeyre, M. \& Maingon, P. Target volume selection and delineation $(T$ and $N$ ) for primary radiation treatment of oral cavity, 
oropharyngeal, hypopharyngeal and laryngeal squamous cell carcinoma. Oral. Oncol. 87, 131-137 (2018).

6. Coussens, L. M. \& Werb, Z. Inflammation and cancer. Nature 420, 860-867 (2002).

7. Trellakis, S. et al. Polymorphonuclear granulocytes in human head and neck cancer: enhanced inflammatory activity, modulation by cancer cells and expansion in advanced disease. Int. J. Cancer 129, 2183-2193 (2011).

8. Lin, D. T., Cohen, S. M., Coppit, G. L. \& Burkey, B. B. Squamous cell carcinoma of the oropharynx and hypopharynx. Otolanyngol. Clin. North Am. 38, 59-74 (2005). viii.

9. $\mathrm{Xu}, \mathrm{X}$. Y. et al. Advances of circular RNAs in carcinoma. Biomed. Pharmacother. 107, 59-71 (2018).

10. Zhang, H. D., Jiang, L. H., Sun, D. W., Hou, J. C. \& Ji, Z. L. CircRNA: a novel type of biomarker for cancer. Breast Cancer 25, 1-7 (2018).

11. $\mathrm{Qu}, \mathrm{S}$. et al. The emerging functions and roles of circular RNAs in cancer. Cancer Lett. 414, 301-309 (2018).

12. Chen, Y., Lin, Y., Shu, Y., He, J. \& Gao, W. Interaction between N(6)-methyladenosine $(\mathrm{m}(6) \mathrm{A})$ modification and noncoding RNAs in cancer. Mol. Cancer 19, 94 (2020)

13. Zhang, $\mathrm{L}$. et al. The role of $\mathrm{N}(6)$-methyladenosine $(\mathrm{m}(6) \mathrm{A})$ modification in the regulation of circRNAs. Mol. Cancer 19, 105 (2020).

14. Xue, L. et al. m(6) A transferase METTL3-induced IncRNA ABHD11-AS1 promotes the Warburg effect of non-small-cell lung cancer. J. Cell Physiol. 236, 2649-2658 (2020).

15. Meng, S. et al. CircRNA: functions and properties of a novel potential biomarker for cancer. Mol. Cancer 16, 94 (2017).

16. Kristensen, L. S., Hansen, T. B., Veno, M. T. \& Kjems, J. Circular RNAs in cancer: opportunities and challenges in the field. Oncogene 37, 555-565 (2018)

17. Zhong, Q., Huang, J., Wei, J. \& Wu, R. Circular RNA CDR1as sponges miR-7-5p to enhance E2F3 stability and promote the growth of nasopharyngeal carcinoma. Cancer Cell Int. 19, 252 (2019).

18. Chen, Z. X. et al. Effects of radiosensitivity and X-ray dose on miR-7 expression in nasopharyngeal carcinoma. Nan Fang Yi Ke Da Xue Xue Bao 30, 1810-1812 (2010).

19. Shuai, M., Hong, J., Huang, D., Zhang, X. \& Tian, Y. Upregulation of circRNA_0000285 serves as a prognostic biomarker for nasopharyngeal carcinoma and is involved in radiosensitivity. Oncol. Lett. 16, 6495-6501 (2018).

20. Cao, S. et al. Novel circular RNA expression profiles reflect progression of patients with hypopharyngeal squamous cell carcinoma. Oncotarget $\mathbf{8}$, 45367-45379 (2017)

21. Imanishi, S. et al. Diffusion-weighted magnetic resonance imaging for predicting and detecting the early response to chemoradiotherapy of advanced esophageal squamous cell carcinoma. Dig. Surg. 30, 240-248 (2013).
22. Vandecaveye, $\mathrm{V}$. et al. Diffusion-weighted magnetic resonance imaging early after chemoradiotherapy to monitor treatment response in head-and-neck squamous cell carcinoma. Int. J. Radiat. Oncol. Biol. Phys. 82, 1098-1107 (2012).

23. Li, H. et al. Therapeutic targeting of circ-CUX1/EWSR1/MAZ axis inhibits glycolysis and neuroblastoma progression. EMBO Mol. Med. 11, e10835 (2019).

24. Zheng, W. et al. Multiple functions and mechanisms underlying the role of METTL3 in human cancers. Front. Oncol. 9, 1403 (2019).

25. Yin, Y. et al. Emerging roles of circRNA in formation and progression of cancer. J. Cancer 10, 5015-5021 (2019).

26. Yi, Y. C., Chen, X. Y., Zhang, J. \& Zhu, J. S. Novel insights into the interplay between $\mathrm{m}(6) \mathrm{A}$ modification and noncoding RNAs in cancer. Mol. Cancer 19 121 (2020).

27. Dai, F. et al. Crosstalk between RNA m(6)A modification and non-coding RNA contributes to cancer growth and progression. Mol. Ther. Nucleic Acids 22, 62-71 (2020).

28. Kristensen, L. S. et al. The biogenesis, biology and characterization of circular RNAs. Nat. Rev. Genet. 20, 675-691 (2019).

29. Zhang, X. et al. Circ-CUX1 accelerates the progression of neuroblastoma via miR-16-5p/DMRT2 axis. Neurochem. Res. 45, 2840-2855 (2020).

30. Greten, F. R. \& Grivennikov, S. I. Inflammation and cancer: triggers, mechanisms, and consequences. Immunity 51, 27-41 (2019).

31. Mantovani, A., Allavena, P., Sica, A. \& Balkwill, F. Cancer-related inflammation. Nature 454, 436-444 (2008).

32. Morrison, W. B. Inflammation and cancer: a comparative view. J. Vet. Intern. Med. 26, 18-31 (2012).

33. Tezal, M. et al. Chronic periodontitis and the incidence of head and neck squamous cell carcinoma. Cancer Epidemiol. Biomark. Prev. 18, 2406-2412 (2009).

34. Bent, R., Moll, L., Grabbe, S. \& Bros, M. Interleukin-1 Beta-A friend or foe in malignancies? Int. J. Mol. Sci. 19, 2155 (2018).

35. Ruan, J., Wang, S. \& Wang, J. Mechanism and regulation of pyroptosismediated in cancer cell death. Chem. Biol. Interact. 323, 109052 (2020).

36. $\mathrm{Hu}$, B. et al. Inflammation-induced tumorigenesis in the colon is regulated by caspase-1 and NLRC4. Proc. Natl Acad. Sci. USA 107, 21635-21640 (2010).

37. Gao, M. et al. Is NLRP3 or NLRP6 inflammasome activation associated with inflammation-related lung tumorigenesis induced by benzo(a)pyrene and lipopolysaccharide? Ecotoxicol. Environ. Saf. 185, 109687 (2019).

38. Chen, G. Y., Liu, M., Wang, F., Bertin, J. \& Nunez, G. A functional role for Nlrp6 in intestinal inflammation and tumorigenesis. J. Immunol. 186, 7187-7194 (2011).

39. Pizato, N. et al. Omega-3 docosahexaenoic acid induces pyroptosis cell death in triple-negative breast cancer cells. Sci. Rep. 8, 1952 (2018).

40. Zitvogel, L., Kepp, O., Galluzzi, L. \& Kroemer, G. Inflammasomes in carcinogenesis and anticancer immune responses. Nat. Immunol. 13, 343-351 (2012). 